\title{
ON THE THEORY OF FROBENIUS EXTENSIONS AND ITS APPLICATION TO LIE SUPERALGEBRAS
}

\author{
ALLEN D. BELL AND ROLF FARNSTEINER
}

\begin{abstract}
By using an approach to the theory of Frobenius extensions that emphasizes notions related to associative forms, we obtain results concerning the trace and corestriction mappings and transitivity. These are employed to show that the extension of enveloping algebras determined by a subalgebra of a Lie superalgebra is a Frobenius extension, and to study certain questions in representation theory.
\end{abstract}

\section{INTRODUCTION}

The theory of Frobenius extensions, initiated independently by Kasch [10] and Nakayama-Tsuzuku [13], has, aside from its intrinsic value, proven to be a useful tool in the study of groups and modular Lie algebras. The twofold purpose of this note is to extend the abstract theory as well as to point out another application concerning the theory of Lie superalgebras.

In $\S 1$ we reformulate some of the basic features of the theory of Frobenius extensions and generalize various results of $[2,15]$, including results on transitivity of Frobenius extensions. Our approach, which emphasizes associative forms, is particularly useful for the study of the trace and corestriction mappings. The second section is devoted to the investigation of extensions defined by the universal enveloping algebras of a Lie superalgebra $L$ and a subalgebra $K$ containing the space $L_{0}$ of even elements. Our main result, namely that their respective enveloping algebras define a Frobenius extension, strikingly parallels recent work on restricted Lie algebras (cf. [3]).

The irreducible modules of the so-called basic classical Lie superalgebras are obtained, according to [8], by inducing from representations of certain subalgebras. By showing that some of the essential features of this theory can be derived from the general principles of the theory of Frobenius extensions, we provide a new conceptual approach to various well-known results.

Frobenius extensions can in particular be used to investigate the cohomology theory of the defining rings. It is, for instance, possible to introduce a complete relative cohomology theory that generalizes well-known principles from the theory of groups (cf. $[4,11])$. We shall not dwell on these aspects here, but rather

Received by the editors October 22, 1990.

1991 Mathematics Subject Classification. Primary 16A36; Secondary 17B70, $17 \mathrm{~B} 35$.

Key words and phrases. Frobenius extensions, Lie superalgebras.

The second author wishes to thank Mr. and Mrs. J. Karam for their hospitality during the writing of a portion of this paper. 
briefly illustrate in $\S 3$ some applications related to more recent developments of the theory.

\section{BASIC RESUlts CONCERNING FrobenIUS EXTENSIONS}

Throughout this paper all rings are assumed to have an identity that operates via the identity transformation on all modules under consideration. Modules are left modules, unless otherwise stated. Let $S$ be a subring of $R$, and suppose that $\alpha$ is an automorphism of $S$. If $M$ is an $S$-module, we let ${ }_{\alpha} M$ denote the $S$-module with a new action defined by $s * m=\alpha(s) m$. Thus, for example, $\operatorname{Hom}_{S}\left(R,{ }_{\alpha} S\right)$ denotes the set of additive maps $f: R \rightarrow S$ such that $f(s r)=$ $\alpha(s) f(r)$ for all $s \in S, r \in R$. This is an $(R, S)$-bimodule via the action $(r \cdot f \cdot s)(x)=f(x r) s$. We say $R$ is an $\alpha$-Frobenius extension of $S$ if

(i) $R$ is a finitely generated projective $S$-module, and

(ii) there exists an isomorphism $\varphi: R \rightarrow \operatorname{Hom}_{S}\left(R,{ }_{\alpha} S\right)$ of $(R, S)$-bimodules.

Such an extension is referred to as free if $R$ is a free $S$-module. In the literature, Frobenius extensions are frequently defined using right modules. Both notions are equivalent in the sense that every left $\alpha$-Frobenius extension is a right $\alpha^{-1}$. Frobenius extension (cf. the remarks after (1.2)).

Given an endomorphism $\beta$ of $S$, a $\beta$-associative form from $R$ to $S$ is a biadditive map $\langle\rangle:, R \times R \rightarrow S$ such that
(a) $\langle s x, y\rangle=s\langle x, y\rangle$,
(b) $\langle x, y s\rangle=\langle x, y\rangle \beta(s)$,
(c) $\langle x r, y\rangle=\langle x, r y\rangle$

for all $s \in S, r, x, y \in R$. We say that $\langle$,$\rangle is nondegenerate if its left and$ right radicals are trivial.

A $\beta$-associative form determines an additive map $\pi: R \rightarrow S ; \pi(r):=$ $\langle r, 1\rangle=\langle 1, r\rangle$ that satisfies $\pi(s r)=s \pi(r)$ and $\pi(r s)=\pi(r) \beta(s)$ for all $r \in R$ and $s \in S$. Conversely, given any additive map $\pi: R \rightarrow S$ with these properties, we can define a $\beta$-associative form from $R$ to $S$ by means of $\langle x, y\rangle:=\pi(x y)$.

In this section we shall provide conditions pertaining to $\alpha^{-1}$-associative forms that are tantamount to an extension of rings being an $\alpha$-Frobenius extension. This approach, which amounts to a refinement of the methods introduced in [15], will be employed later to show that enveloping algebras of Lie superalgebras give rise to Frobenius extensions.

Definition. Let $\langle\rangle:, R \times R \rightarrow S$ be an $\alpha^{-1}$-associative form. We say that two finite subsets $\left\{x_{1}, \ldots, x_{n}\right\}$ and $\left\{y_{1}, \ldots, y_{n}\right\}$ of $R$ form a dual projective pair relative to $\langle$,$\rangle if$

$$
r=\sum_{i=1}^{n} y_{i} \alpha\left(\left\langle x_{i}, r\right\rangle\right)=\sum_{i=1}^{n}\left\langle r, y_{i}\right\rangle x_{i} \quad \text { for all } r \in R .
$$

Note that the $S$-linear mappings $\varphi_{i}: R \rightarrow S ; \varphi_{i}(r):=\left\langle r, y_{i}\right\rangle$ together with the set $\left\{x_{1}, \ldots, x_{n}\right\}$ form a projective basis for $R$ as an $S$-module.

Let $\left\{x_{1}, \ldots, x_{n}\right\}$ and $\left\{y_{1}, \ldots, y_{n}\right\}$ be subsets of $R$ with $R=\sum_{i=1}^{n} S x_{i}=$ $\sum_{i=1}^{n} y_{i} S$. In the ring $M_{n}(S)$ of $(n \times n)$ matrices with coefficients in $S$, define $A=\left[\left\langle x_{i}, y_{j}\right\rangle\right]_{1 \leq i, j \leq n}$. If $\left\{x_{1}, \ldots, x_{n}\right\}$ and $\left\{y_{1}, \ldots, y_{n}\right\}$ form a dual projective pair relative to the $\alpha^{-1}$-associative form $\langle$,$\rangle , then the i, j$-entry of $A^{2}$ 
is

$$
\sum_{k=1}^{n}\left\langle x_{i}, y_{k}\right\rangle\left\langle x_{k}, y_{j}\right\rangle=\left\langle\sum_{k=1}^{n}\left\langle x_{i}, y_{k}\right\rangle x_{k}, y_{j}\right\rangle=\left\langle x_{i}, y_{j}\right\rangle,
$$

proving that $A$ is idempotent. In addition, if $r \in R$ and $\langle r, R\rangle=0$, then $r=\sum_{i=1}^{n}\left\langle r, y_{i}\right\rangle x_{i}=0$. Likewise $\langle R, r\rangle=0$ implies $r=0$, so $\langle$,$\rangle is$ nondegenerate.

Suppose conversely that $A$ is idempotent and $\langle$,$\rangle is nondegenerate. The$ above calculation shows $\left\langle x_{i}-\sum_{k=1}^{n}\left\langle x_{i}, y_{k}\right\rangle x_{k}, y_{j}\right\rangle=0$ for all $j$. Since $R=$ $\sum_{j=1}^{n} y_{j} S$, the element $x_{i}-\sum_{k=1}^{n}\left\langle x_{i}, y_{k}\right\rangle x_{k}$ is contained in the left radical of $\langle$,$\rangle . Consequently, x_{i}=\sum_{k=1}^{n}\left\langle x_{i}, y_{k}\right\rangle x_{k}$ for all $i$, and since $\sum_{i=1}^{n} S x_{i}=R$, this implies $r=\sum_{k=1}^{n}\left\langle r, y_{k}\right\rangle x_{k}$ for any $r \in R$. By the same token, we have $r=$ $\sum_{k=1}^{n} y_{k} \alpha\left(\left\langle x_{k}, r\right\rangle\right)$, so $\left\{x_{1}, \ldots, x_{n}\right\}$ and $\left\{y_{1}, \ldots, y_{n}\right\}$ form a dual projective pair.

Theorem 1.1. Let $S$ be a subring of $R$ and suppose that $\alpha$ is an automorphism of $S$. Then the following statements are equivalent.

(a) $R$ is an $\alpha$-Frobenius extension of $S$.

(b) There is an $\alpha^{-1}$-associative form $\langle$,$\rangle from R$ to $S$ relative to which a dual projective pair $\left\{x_{1}, \ldots, x_{n}\right\},\left\{y_{1}, \ldots, y_{n}\right\}$ exists.

(c) There exist a nondegenerate $\alpha^{-1}$-associative form $\langle$,$\rangle from R$ to $S$ and two subsets $\left\{x_{1}, \ldots, x_{n}\right\}$ and $\left\{y_{1}, \ldots, y_{n}\right\}$ of $R$ with $R=\sum_{i=1}^{n} S x_{i}=$ $\sum_{i=1}^{n} y_{i} S$ such that the matrix $A=\left[\left\langle x_{i}, y_{j}\right\rangle\right]_{i, j}$ is equivalent to an idempotent matrix in the sense that there is a left invertible $P \in M_{n}(S)$ and a right invertible $Q \in M_{n}(S)$ with $P A Q$ idempotent.

Proof. (a) $\Rightarrow$ (b) Let $\varphi: R \rightarrow \operatorname{Hom}_{S}\left(R,{ }_{\alpha} S\right)$ be the defining isomorphism of $(R, S)$-bimodules. We define a bilinear form $\langle\rangle:, R \times R \rightarrow S$ by means of $\langle x, y\rangle:=\alpha^{-1}(\varphi(y)(x))$. Using the definition of the bimodule actions, one readily verifies that $\langle$,$\rangle is an \alpha^{-1}$-associative form.

Let $x_{1}, \ldots, x_{n} \in R$ and $\varphi_{1}, \ldots, \varphi_{n} \in \operatorname{Hom}_{S}(R, S)$ form a projective basis for the $S$-module $R$. Since $\alpha \circ \varphi_{i} \in \operatorname{Hom}_{S}\left(R,{ }_{\alpha} S\right)$, there exist elements $y_{i}$ of $R$ for $1 \leq i \leq n$ such that $\varphi\left(y_{i}\right)=\alpha \circ \varphi_{i}$. Now

$$
r=\sum_{i=1}^{n} \varphi_{i}(r) x_{i}=\sum_{i=1}^{n} \alpha^{-1}\left(\varphi\left(y_{i}\right)(r)\right) x_{i}=\sum_{i=1}^{n}\left\langle r, y_{i}\right\rangle x_{i}
$$

for any $r \in R$. Applying $f \in \operatorname{Hom}_{S}\left(R,{ }_{\alpha} S\right)$ to this last equation shows that

$$
f=\sum_{i=1}^{n} \varphi\left(y_{i}\right) \cdot f\left(x_{i}\right) \text {. }
$$

If $f=\varphi(r)$, this gives $\varphi(r)=\varphi\left(\sum_{i=1}^{n} y_{i} \alpha\left(\left\langle x_{i}, r\right\rangle\right)\right)$, whence $r=\sum_{i=1}^{n} y_{i} \alpha\left(\left\langle x_{i}, r\right\rangle\right)$ for any $r \in R$.

The implication $(\mathrm{b}) \Rightarrow(\mathrm{c})$ is a direct consequence of the remarks preceding the theorem. To verify $(\mathrm{c}) \Rightarrow$ (a) we define $a_{1}, \ldots, a_{n}, b_{1}, \ldots, b_{n} \in R$ by $\left(a_{1}, \ldots, a_{n}\right)^{t}=P\left(x_{1}, \ldots, x_{n}\right)^{t}$ and $\left(b_{1}, \ldots, b_{n}\right)=\left(y_{1}, \ldots, y_{n}\right) \alpha(Q)$. The left invertibility of $P$ and the right invertibility of $\alpha(Q)$ ensure that $R=\sum_{i=1}^{n} S a_{i}=\sum_{i=1}^{n} b_{i} S$. Clearly $\left[\left\langle a_{i}, b_{j}\right\rangle\right]_{1 \leq i, j \leq n}=P A Q$, and so by the remarks preceding the theorem $\left\{a_{1}, \ldots, a_{n}\right\}$ and $\left\{b_{1}, \ldots, b_{n}\right\}$ form a dual projective pair. Define an $(R, S)$-bimodule map $\varphi: R \rightarrow \operatorname{Hom}_{S}\left(R,{ }_{\alpha} S\right)$ by 
$\varphi(y)(x):=\alpha(\langle x, y\rangle)$. To prove the invertibility of $\varphi$ we consider the mapping $\psi: \operatorname{Hom}_{S}\left(R,{ }_{\alpha} S\right) \rightarrow R ; \psi(f):=\sum_{i=1}^{n} b_{i} f\left(a_{i}\right)$. For any $r \in R$, we have

$$
\psi(\varphi(r))=\sum_{i=1}^{n} b_{i} \varphi(r)\left(a_{i}\right)=\sum_{i=1}^{n} b_{i} \alpha\left(\left\langle a_{i}, r\right\rangle\right)=r .
$$

Similarly, we obtain for $f \in \operatorname{Hom}_{S}\left(R,{ }_{\alpha} S\right)$ :

$$
\begin{aligned}
\varphi(\psi(f))(r) & =\alpha(\langle r, \psi(f)\rangle)=\sum_{i=1}^{n} \alpha\left(\left\langle r, b_{i} f\left(a_{i}\right)\right\rangle\right) \\
& =\sum_{i=1}^{n} \alpha\left(\left\langle r, b_{i}\right\rangle\right) f\left(a_{i}\right)=f\left(\sum_{i=1}^{n}\left\langle r, b_{i}\right\rangle a_{i}\right)=f(r) .
\end{aligned}
$$

Thus $\varphi$ and $\psi$ are inverses, proving that (a) holds.

Let $R: S$ be an $\alpha$-Frobenius extension, let $\langle$,$\rangle be the \alpha^{-1}$-associative form from $R$ to $S$ in (1.1), and define $\pi: R \rightarrow S$ by $\pi(r):=\langle r, 1\rangle=\langle 1, r\rangle$. The maps $\alpha \circ \pi$ and $\pi$ are customarily referred to as the Frobenius homomorphisms associated to this Frobenius extension. In this paper we shall only refer to $\pi$ as the Frobenius homomorphism. Our remarks above show the form $\langle$,$\rangle is$ determined by $\pi$.

Definition. Let $\langle\rangle:, R \times R \rightarrow S$ be an $\alpha^{-1}$-associative form. Two subsets $\left\{x_{1}, \ldots, x_{n}\right\}$ and $\left\{y_{1}, \ldots, y_{n}\right\}$ of $R$ are said to form a dual free pair relative to $\langle$,$\rangle if$

(i) $R=\sum_{i=1}^{n} S x_{i}=\sum_{i=1}^{n} y_{i} S$,

(ii) $\left\langle x_{i}, y_{j}\right\rangle=\delta_{i j}$ for $1 \leq i, j \leq n$.

Note that $\left\{x_{1}, \ldots, x_{n}\right\}$ and $\left\{y_{1}, \ldots, y_{n}\right\}$ are bases for $R$ as a left and right $S$-module, respectively. A direct computation shows that $\left\{x_{1}, \ldots, x_{n}\right\}$ and $\left\{y_{1}, \ldots, y_{n}\right\}$ form a dual free pair if and only if they form a dual projective pair and they are bases for $R$ as a left and right $S$-module, respectively.

The analogue of (1.1) for free Frobenius extensions is the following.

Corollary 1.2. Let $S$ be a subring of $R$ and let $\alpha$ be an automorphism of $S$. Then the following statements are equivalent.

(a) $R$ is a free $\alpha$-Frobenius extension of $S$.

(b) There is an $\alpha^{-1}$-associative form $\langle$, $\rangle$ from $R$ to $S$ relative to which a dual free pair $\left\{x_{1}, \ldots, x_{n}\right\},\left\{y_{1}, \ldots, y_{n}\right\}$ exists.

(c) There exist an $\alpha^{-1}$-associative form $\langle$, $\rangle$ from $R$ to $S$ and elements $x_{1}, \ldots, x_{n}, y_{1}, \ldots, y_{n} \in R$ such that $R=\sum_{i=1}^{n} S x_{i}=\sum_{i=1}^{n} y_{i} S$ and the $n \times n$ matrix $\left[\left\langle x_{i}, y_{j}\right\rangle\right]_{i, j}$ has a two-sided inverse in the ring $M_{n}(S)$.

Proof. (a) $\Rightarrow$ (b) Define an $\alpha^{-1}$-associative bilinear form $\langle\rangle:, R \times R \rightarrow S$ as in the proof of (1.1). Let $\left\{x_{1}, \ldots, x_{n}\right\}$ be a basis of the left $S$-module $R$. Since $\varphi$ is surjective there exist $y_{1}, \ldots, y_{n}$ in $R$ such that $\alpha^{-1} \circ \varphi\left(y_{j}\right)$ is the $j$ th projection from $R$ onto $S$ relative to this basis. Clearly $\left\langle x_{i}, y_{j}\right\rangle=\delta_{i j}$ for $1 \leq i, j \leq n$. Since $\left\{y_{1}, \ldots, y_{n}\right\}$ generates the right $S$-module $R$ as in the proof of (1.1), we obtain (b).

The implication $(\mathrm{b}) \Rightarrow(\mathrm{c})$ is trivial. As regards $(\mathrm{c}) \Rightarrow(\mathrm{a})$, it follows directly from the invertibility of $\left[\left\langle x_{i}, y_{j}\right\rangle\right]$ that the form $\langle$,$\rangle is nondegenerate. An$ application of (1.1) now yields the desired result. 
Examples. (A) If $S$ is any ring and $R=M_{n}(S)$, define $\langle x, y\rangle:=\operatorname{Tr}(x y)$. If we let $e_{i j}$ denote the matrix with 1 in the $i, j$ position and 0's elsewhere, then $\left\{e_{i j}\right\}_{1 \leq i, j \leq n}$ and $\left\{e_{j i}\right\}_{1 \leq i, j \leq n}$ form a dual free pair. Thus $R$ is a free Frobenius extension of $S$ by $(1.2(\mathrm{~b}))$.

(B) It is well known that if $G$ is a group and $H$ is a subgroup of finite index, the group ring $K[G]$ is a free Frobenius extension of $K[H]$. This result can be generalized to the case of any strongly $G$-graded ring (for example a crossed product) in place of $K[G]$. Recall that a ring $R$ is graded by $G$ if $R=$ $\bigoplus_{g \in G} R_{g}$, where each $R_{g}$ is an Abelian subgroup of $R$ and $R_{g} R_{h} \subseteq R_{g h}$ for all $g, h \in G$. We say $R$ is strongly graded by $G$ if equality always holds. This condition is easily seen to be equivalent to the existence of $x_{1, g}, \ldots, x_{m(g), g} \in$ $R_{g}, y_{1, g}, \ldots, y_{m(g), g} \in R_{g^{-1}}$ with $\sum_{i=1}^{m(g)} y_{i, g} x_{i, g}=1$ for each $g \in G$. If $R$ is strongly graded by $G$ and $S=\bigoplus_{g \in H} R_{g}$, then $R: S$ is an $\operatorname{id}_{S}$-Frobenius extension.

To see this, we define the Frobenius homomorphism by $\pi\left(\sum_{g \in G} r_{g}\right):=$ $\sum_{g \in H} r_{g}$. If we let $\{H g: g \in C\}$ be a complete set of right cosets for $H$ in $G$, then it is clear that $\left\{x_{i, g}: g \in C, 1 \leq i \leq m(g)\right\}$ and $\left\{y_{i, g}: g \in\right.$ $C, 1 \leq i \leq m(g)\}$ form a dual projective pair.

(C) We give an example which employs a method very much like our proof in $\S 2$ that enveloping algebras of Lie superalgebras give rise to Frobenius extensions. Let $S$ be a subring of $R$ and suppose that there exist an $x \in R$, a positive integer $m$, and an automorphism $\alpha$ of $S$ such that

(i) $1, x, \ldots, x^{m}$ is a basis for $R$ as a left $S$-module, and

(ii) $s x-x \alpha(s) \in S$ for all $s \in S$.

Then $R: S$ is a free $\alpha^{m}$-Frobenius extension.

To see this, first note that we can use (ii) and induction to prove that for any positive integer $k$,

(*) $s x^{k}-x^{k} \alpha^{k}(s) \in S+x S+\cdots+x^{k-1} S=S+S x+\cdots+S x^{k-1}$ for all $s \in S$.

Thus $1, x, \ldots, x^{m}$ is also a basis for $R$ as a right $S$-module. Now define $\pi: R \rightarrow S$ by $\pi\left(\sum_{i=0}^{m} s_{i} x^{i}\right):=s_{m}$. Clearly $\pi$ is left $S$-linear, and (*) implies that for any $s \in S$, there exist $s_{0}^{\prime}, \ldots, s_{m-1}^{\prime} \in S$ such that $\sum_{i=0}^{m} s_{i} x^{i} s=$ $s_{m} \alpha^{-m}(s) x^{m}+\sum_{i=0}^{m-1} s_{i}^{\prime} x^{i}$. It follows that the form $\langle\rangle:, R \times R \rightarrow S ;\left\langle r_{1}, r_{2}\right\rangle:=$ $\pi\left(r_{1} r_{2}\right)$, is $\left(\alpha^{m}\right)^{-1}$-associative.

If $0 \leq i<j \leq m$, then $\left\langle x^{i}, x^{m-j}\right\rangle=0$, while $\left\langle x^{i}, x^{m-i}\right\rangle=1$. Consequently the matrix $\left[\left\langle x^{i}, x^{m-j}\right\rangle\right]_{0 \leq i, j \leq m}$ is a lower triangular matrix all of whose diagonal entries are 1. Such a matrix is invertible, and so $R: S$ is a free $\alpha^{m}$-Frobenius extension by (1.2).

(D) As a special case of example (C), let $K$ be any ring and consider the extension $R: S$ given by $R=M_{2}(K)$, and $S=\left\{\left[\begin{array}{ll}a & b \\ 0 & a\end{array}\right]: a, b \in K\right\}$. This satisfies the hypotheses of $(\mathrm{C})$ if we set $x=\left[\begin{array}{ll}0 & 0 \\ 1 & 0\end{array}\right]$ and define $\alpha\left(\left[\begin{array}{ll}a & b \\ 0 & a\end{array}\right]\right):=\left[\begin{array}{cc}a & -b \\ 0 & a\end{array}\right]$. Thus $R: S$ is a free $\alpha$-Frobenius extension. (The Frobenius homomorphism in this case is defined by $\pi\left(\left[\begin{array}{ll}a & b \\ c & d\end{array}\right]\right)=\left[\begin{array}{cc}c & a-d \\ 0 & c\end{array}\right]$. If we were to define $\pi^{\prime}: R \rightarrow S$ by $\pi^{\prime}\left(\left[\begin{array}{ll}a & b \\ c & d\end{array}\right]\right)=\left[\begin{array}{cc}c & a+d \\ 0 & c\end{array}\right]$, then $R: S$ could be shown to be an $\mathrm{id}_{S}$-Frobenius extension as well.)

One interesting facet of this example is that $S \cong K[t] /\left(t^{2}\right)$, where $t$ is an 
indeterminate, so the global dimension $\operatorname{gl} \operatorname{dim} S$ is infinite, while $\operatorname{gl} \operatorname{dim} R=$ gl.dim $K$. Since $R: S$ is a Frobenius extension, the relative global dimension of $R: S$ is 0 or $\infty$. Consequently, this example shows no simple relation need hold between the actual global dimensions of $R$ and $S$. In addition, if we take $K$ of finite global dimension, we obtain an example of a finite free ring extension where the extension ring has smaller global dimension than the base ring. This is a simpler example than those usually cited in the literature, as well as being valid in any characteristic (cf. [12, 7.2.7]).

(E) If we let $K$ be a field and $V$ a vector space of dimension $n$, then one can easily iterate example $(C)$ and show that the Grassmann algebra $\Lambda(V)$ is a Frobenius algebra over $K$, with the Frobenius homomorphism $\pi: R \rightarrow K$ determined by the projection onto $\Lambda^{n}(V)$.

Remarks. (1) If $R: S$ is an $\alpha$-Frobenius extension with form $\langle$,$\rangle , then$ one readily sees from (1.1) that the extension defined by their opposite rings is an $\alpha^{-1}$-Frobenius extension with defining form $(x, y):=\alpha(\langle y, x\rangle)$. This in particular implies the well-known fact that Frobenius extensions can be equally well defined in terms of right modules.

(2) Suppose $R_{i}$ is an $\alpha_{i}$-Frobenius extension of $S_{i}$ for $1 \leq i \leq n$, and each $S_{i}$ and each $R_{i}$ is an algebra over the commutative ring $K$, and every $\alpha_{i}$ is $K$ linear. Then the tensor product over $K$ of the $R_{i}$ is an $\alpha$-Frobenius extension of the tensor product over $K$ of the $S_{i}$, where $\alpha$ is the tensor product of the $\alpha_{i}$. This is easy to see using (1.1): a dual projective pair for $R: S$ is obtained by tensoring together the elements of the dual projective pairs for the extensions $R_{i}: S_{i}$.

It now readily follows that if $R: S$ is an $\alpha$-Frobenius extension, then $R \otimes_{\mathbb{Z}}$ $R^{\text {op }}$ is an $\left(\alpha \otimes \alpha^{-1}\right)$-Frobenius extension of $S \otimes_{\mathbb{Z}} S^{\text {op }}$. This enables us to apply module-theoretic results on Frobenius extensions to bimodules.

Now let $R: S$ be an $\alpha$-Frobenius extension and consider two $R$-modules $M, N$. Given a dual projective pair $\left\{x_{1}, \ldots, x_{n}\right\},\left\{y_{1}, \ldots, y_{n}\right\}$ the mapping $\operatorname{Tr}_{[R: S]}: \operatorname{Hom}_{S}\left(M,{ }_{\alpha} N\right) \rightarrow \operatorname{Hom}_{R}(M, N)$, which is defined by

$$
\operatorname{Tr}_{[R: S]}(f)(m)=\sum_{i=1}^{n} y_{i} f\left(x_{i} m\right) \quad \text { for } m \in M
$$

is customarily referred to as the trace map.

The ensuing result elaborates on [15, Satz 6] by providing a factorization of the trace map.

Proposition 1.3. Let $T \subset S \subset R$ be rings such that

(a) $R: S$ is an $\alpha$-Frobenius extension,

(b) $S: T$ is a $\gamma$-Frobenius extension, and

(c) $\alpha(T)=T$.

Then $R: T$ is an $(\alpha \circ \gamma)$-Frobenius extension and $\operatorname{Tr}_{[R: T]}=\operatorname{Tr}_{[R: S]} \circ \operatorname{Tr}_{[S: T]}$. Proof. Let $\pi: R \rightarrow S$ and $\rho: S \rightarrow T$ denote the Frobenius homomorphisms of $R: S$ and $S: T$, respectively. We define an $(\alpha \circ \gamma)^{-1}$-associative form $\langle\rangle:, R \times R \rightarrow T$ via $\langle a, b\rangle:=(\rho \circ \pi)(a b)$ for $a, b \in R$. The extensions $R: S$ and $S: T$ have associated dual projective pairs $\left\{x_{1}, \ldots, x_{n}\right\},\left\{y_{1}, \ldots, y_{n}\right\}$ and $\left\{u_{1}, \ldots, u_{m}\right\},\left\{v_{1}, \ldots, v_{m}\right\}$, respectively. Then, for every element $r$ 
of $R$,

$$
\begin{aligned}
r & =\sum_{i=1}^{n} y_{i} \alpha\left(\pi\left(x_{i} r\right)\right)=\sum_{i=1}^{n} y_{i} \alpha\left(\sum_{j=1}^{m} v_{j} \gamma\left(\rho\left(u_{j} \pi\left(x_{i} r\right)\right)\right)\right) \\
& =\sum_{i=1}^{n} \sum_{j=1}^{m} y_{i} \alpha\left(v_{j}\right)(\alpha \circ \gamma)\left((\rho \circ \pi)\left(u_{j} x_{i} r\right)\right) \\
& =\sum_{i, j} y_{i} \alpha\left(v_{j}\right)(\alpha \circ \gamma)\left(\left\langle u_{j} x_{i}, r\right\rangle\right) .
\end{aligned}
$$

Similarly, $r=\sum_{i, j}\left\langle r, y_{i} \alpha\left(v_{j}\right)\right\rangle u_{j} x_{i}$. This shows that $\left\{u_{j} x_{i}: 1 \leq i \leq n, 1 \leq\right.$ $j \leq m\}$ and $\left\{y_{i} \alpha\left(v_{j}\right): 1 \leq i \leq n, 1 \leq j \leq m\right\}$ form a dual projective pair relative to $\langle$,$\rangle . According to (1.1), R: T$ is therefore an $(\alpha \circ \gamma)$-Frobenius extension.

Now let $f$ be an element of $\operatorname{Hom}_{T}\left(M,{ }_{\alpha \circ \gamma} N\right)=\operatorname{Hom}_{T}\left(M,{ }_{\gamma}\left({ }_{\alpha} N\right)\right)$. Then $\operatorname{Tr}_{[S: T]}(f) \in \operatorname{Hom}_{S}\left(M,{ }_{\alpha} N\right)$ and

$$
\operatorname{Tr}_{[S: T]}(f)(m)=\sum_{j=1}^{m} v_{j} * f\left(u_{j} m\right)=\sum_{j=1}^{m} \alpha\left(v_{j}\right) f\left(u_{j} m\right) .
$$

Consequently,

$$
\begin{gathered}
\operatorname{Tr}_{[R: S]} \circ \operatorname{Tr}_{[S: T]}(f)(m)=\sum_{i=1}^{n} y_{i} \operatorname{Tr}_{[S: T]}(f)\left(x_{i} m\right) \\
=\sum_{i, j} y_{i} \alpha\left(v_{j}\right) f\left(u_{j} x_{i} m\right)=\operatorname{Tr}_{[R: T]}(f)(m) .
\end{gathered}
$$

The following result provides a partial converse to (1.3).

Theorem 1.4. Let $T \subset S \subset R$ be rings and suppose that

(a) $R: T$ is a $\beta$-Frobenius extension with Frobenius homomorphism $\sigma: R \rightarrow$ $T$,

(b) $S: T$ is a $\gamma$-Frobenius extension with Frobenius homomorphism $\rho: S \rightarrow$ $T$, and

(c) $R$ is a projective $S$-module.

Then the following statements hold:

(1) The map $\pi:=\operatorname{Tr}_{[S: T]}(\gamma \circ \sigma)$ is the unique element of $\operatorname{Hom}_{S}(R, S)$ such that $\rho \circ \pi=\sigma$.

(2) If there exists an automorphism $\alpha$ of $S$ with $\pi(r s)=\pi(r) \alpha^{-1}(s)$ for all $r \in R, s \in S$, then $R: S$ is an $\alpha$-Frobenius extension with Frobenius homomorphism $\pi$.

Proof. Let $\left\{x_{1}, \ldots, x_{n}\right\},\left\{y_{1}, \ldots, y_{n}\right\}$ be a dual projective pair for $S: T$, so that $s=\sum_{i=1}^{n} y_{i} \gamma \circ \rho\left(x_{i} s\right)=\sum_{i=1}^{n} \rho\left(s y_{i}\right) x_{i}$ for all $s \in S$.

(1) Suppose that $\pi=\operatorname{Tr}_{[S: T]}(\gamma \circ \sigma)$. For $r \in R$ we obtain

$$
\begin{aligned}
(\rho \circ \pi)(r) & =\rho\left(\sum_{i=1}^{n} y_{i}(\gamma \circ \sigma)\left(x_{i} r\right)\right)=\sum_{i=1}^{n} \rho\left(y_{i}\right) \sigma\left(x_{i} r\right) \\
& =\sigma\left(\sum_{i=1}^{n} \rho\left(y_{i}\right) x_{i} r\right)=\sigma(r),
\end{aligned}
$$


so $\rho \circ \pi=\sigma$. Assume conversely that $\rho \circ \pi=\sigma$. As $\operatorname{Tr}_{[S: T]}(\gamma \circ \rho)=\mathrm{id}_{S}$, it follows that

$$
\operatorname{Tr}_{[S: T]}(\gamma \circ \sigma)=\operatorname{Tr}_{[S: T]}(\gamma \circ \rho \circ \pi)=\operatorname{Tr}_{[S: T]}(\gamma \circ \rho) \circ \pi=\pi .
$$

(2) Define $\varphi: R \rightarrow \operatorname{Hom}_{S}\left(R,{ }_{\alpha} S\right)$ by $\varphi(r)=r \cdot(\alpha \circ \pi)$, i.e., $\varphi(r)\left(r^{\prime}\right)=$ $\alpha\left(\pi\left(r^{\prime} r\right)\right)$. Our assumption concerning $\pi$ ensures that $\varphi$ is a well-defined $(R, S)$-bimodule map.

Suppose $r \in \operatorname{Ker} \varphi$. Then for every $r^{\prime} \in R$, we have $\pi\left(r^{\prime} r\right)=0$, whence $0=(\rho \circ \pi)\left(r^{\prime} r\right)=\sigma\left(r^{\prime} r\right)$ for all $r^{\prime} \in R$. Since $R: T$ is a Frobenius extension, it follows that $r=0$.

Suppose $f \in \operatorname{Hom}_{S}\left(R,{ }_{\alpha} S\right)$. Then $\beta \circ \rho \circ \alpha^{-1} \circ f \in \operatorname{Hom}_{T}\left(R,{ }_{\beta} T\right)$; since $R: T$ is a $\beta$-Frobenius extension, there is an $r \in R$ with $\beta \circ \rho \circ \alpha^{-1} \circ f=$ $r \cdot(\beta \circ \sigma)=\beta \circ \rho \circ(r \cdot \pi)$. We can cancel $\beta$ and conclude $\rho \circ \alpha^{-1} \circ f=\rho \circ(r \cdot \pi)$. Hence

$$
\begin{aligned}
r \cdot \pi & =\operatorname{Tr}_{[S: T]}(\gamma \circ \rho) \circ(r \cdot \pi)=\operatorname{Tr}_{[S: T]}(\gamma \circ \rho \circ(r \cdot \pi)) \\
& =\operatorname{Tr}_{[S: T]}\left(\gamma \circ \rho \circ \alpha^{-1} \circ f\right)=\alpha^{-1} \circ f,
\end{aligned}
$$

proving that $\varphi(r)=r \cdot(\alpha \circ \pi)=f$. This shows $\varphi$ is an isomorphism.

The automorphism $\alpha$ in part (2) of the preceding result is uniquely determined. Moreover, its existence is related to the behavior of the Nakayama automorphism of the extension $R: T$. To see this, we first observe that by definition of $\pi$, we have

$$
\pi(r t)=\pi(r) \gamma\left(\beta^{-1}(t)\right)
$$

for any $t \in T, r \in R$. Consequently, $\alpha(t)=\left(\beta \circ \gamma^{-1}\right)(t)$. In particular, $\alpha(T)=T$, and hence $\alpha\left(C_{S}(T)\right)=C_{S}(T)$.

Associated to any Frobenius extension is the Nakayama automorphism, an automorphism of the centralizer of the subring in the extension ring (cf. [11]). In the situation of (1.4), there are two Nakayama automorphisms, $\mu: C_{R}(T) \rightarrow$ $C_{R}(T)$ and $\nu: C_{S}(T) \rightarrow C_{S}(T)$, whose defining relations are

$$
\sigma(c r)=\sigma(r \mu(c)) \text { and } \rho(c s)=\rho(s \nu(c)) .
$$

Suppose that (a) through (c) of (1.4) hold and put $\pi:=\operatorname{Tr}_{[S: T]}(\gamma \circ \sigma)$. Then the following statements are equivalent:

(i) $\mu\left(C_{S}(T)\right)=C_{S}(T)$.

(ii) There exists an automorphism $\alpha$ of $C_{S}(T)$ such that $\pi(r s)=\pi(r) \alpha^{-1}(s)$ for all $r \in R, s \in C_{S}(T)$.

To prove (i) implies (ii), suppose that $\mu\left(C_{S}(T)\right)=C_{S}(T)$. Then for any $r \in R, c \in C_{S}(T)$, we have

$$
\rho \circ \pi(r c)=\sigma(r c)=\sigma\left(\mu^{-1}(c) r\right)=\rho\left(\mu^{-1}(c) \pi(r)\right)=\rho\left(\pi(r)\left(\nu \circ \mu^{-1}\right)(c)\right) .
$$

Upon composing both sides with $\gamma$ and applying the operator $\operatorname{Tr}_{[S: T]}$, we obtain $\pi(r c)=\pi(r)\left(\nu \circ \mu^{-1}\right)(c)$. Consequently, $\alpha:=\mu \circ \nu^{-1}$ has the requisite property.

Conversely, assume (ii) holds. For $c \in C_{S}(T)$ and $r \in R$ we obtain

$$
\begin{aligned}
\sigma\left(\mu^{-1}(c) r\right) & =\rho \circ \pi(r c)=\rho\left(\pi(r) \alpha^{-1}(c)\right)=\rho\left(\left(\nu^{-1} \circ \alpha^{-1}\right)(c) \pi(r)\right) \\
& =\rho \circ \pi\left(\left(\nu^{-1} \circ \alpha^{-1}\right)(c) r\right)=\sigma\left(\left(\nu^{-1} \circ \alpha^{-1}\right)(c) r\right) .
\end{aligned}
$$


Consequently, $\mu^{-1}(c)=\left(\nu^{-1} \circ \alpha^{-1}\right)(c)$. As a result we have $\mu\left(C_{S}(T)\right)=C_{S}(T)$.

It follows from our above deliberations that condition (i) is necessary for the existence of $\alpha$ in (1.4)(2).

If $T$ is contained in the center of $S$, the above calculations show that $\pi(r s)=\pi(r)\left(\nu \circ \mu^{-1}(s)\right)$ for all $r \in R, s \in S$. Thus, in this case (1.4) can be restated as follows.

Corollary 1.5 [15, Satz 7]. Let $T \subset S \subset R$ be rings such that $T \subset Z(S)$ and suppose that the following conditions hold.

(a) $R: T$ is a $\beta$-Frobenius extension with Nakayama automorphism $\mu$ : $C_{R}(T) \rightarrow C_{R}(T)$ such that $\mu(S)=S$,

(b) $S: T$ is a Frobenius extension with Nakayama automorphism $\nu: S \rightarrow$ $S$, and

(c) $R$ is a projective $S$-module.

Then $R: S$ is a $\left(\mu \circ \nu^{-1}\right)$-Frobenius extension.

Examples. We give an example showing that the technical assumption (c) is essential to the validity of (1.4) and (1.5).

(F) Let $K$ be a field and $t$ an indeterminate, and set $R:=K[t] /\left(t^{3}\right)=K[\bar{t}]$. Let $S$ be the subalgebra $K\left[\bar{t}^{2}\right]$ of $R$, and set $T:=K$. Then by example (C), $R$ and $S$ are Frobenius algebras with $\sigma: R \rightarrow K$ given by $\sigma\left(a+b \bar{t}+c \bar{t}^{2}\right)=c$ and $\rho: S \rightarrow K$ obtained by restricting $\sigma$ to $S$. All the hypotheses of (1.4) and (1.5) are satisfied in this setting except projectivity of $R$ as an $S$-module. Since $S$ is a local ring, if $R$ were projective, it would be free. This is impossible, however, since $R$ is 3-dimensional over $K$ and $S$ is 2-dimensional. (In fact, one can show in this example that $\operatorname{Hom}_{S}(R, S) \cong R$, so $R: S$ satisfies all the conditions for a Frobenius extension except projectivity.)

The following example illustrates that Frobenius extensions also arise if the technical assumptions of (1.4) and (1.5) do not hold.

(G) Let $K$ be a field whose characteristic is not 2 and let $R$ be the Grassmann algebra over $K$ in the indeterminates $x, y$. By example $(\mathrm{E}), R$ is a Frobenius algebra over $K$ with Frobenius homomorphism $\sigma: R \rightarrow K$ defined by $\sigma(a+b x+c y+d x y)=d$ for $a, b, c, d \in K$. The Nakayama automorphism $\mu$ of $R$ is given by

$$
\mu(a+b x+c y+d x y)=a-b x-c y+d x y .
$$

Set $t:=x+x y \in R$; then $t^{2}=0$, whence $S:=K+K t$ is a subalgebra of $R$. By example $(\mathrm{C}), S$ is also a Frobenius algebra over $K$ with Frobenius homomorphism $\rho: S \rightarrow K$ defined by $\rho(a+b t)=b$. It is easy to see that $\{1, y\}$ is a basis for $R$ as a right or left $S$-module. If we define an automorphism $\alpha$ of $S$ by $\alpha(t)=-t$, then $s y=y \alpha(s)$ for $s \in S$. It thus follows from example (C) that $R: S$ is an $\alpha$-Frobenius extension with Frobenius homomorphism $\pi(a+b x+c y+d x y)=c+(d-b) t$.

Since $S$ is not invariant under the Nakayama automorphism $\mu$ of $R$, the hypothesis $\mu(S)=S$ in (1.5) is independent of the other hypotheses of (1.5), and so by the equivalence of (i) and (ii) in the remarks after (1.4), the hypothesis (2) of (1.4) is independent of the other hypotheses in (1.4). Moreover, this example shows the hypotheses of (1.4) and (1.5) are not necessary for $R: S$ to be a Frobenius extension. 


\section{FROBENIUS EXTENSIONS OF LIE SUPERALGEBRAS}

Let $F$ be a field whose characteristic is not 2 and suppose that $L$ is a Lie superalgebra over $F$ with even part $L_{0}$ and odd part $L_{1}$. (See [8] for definitions and terminology.) We shall be concerned with subalgebras $K=$ $K_{0} \oplus K_{1}$ of $L$ for which $K_{0}$ coincides with $L_{0}$. Note that these subalgebras correspond to the $L_{0}$-submodules of $L_{1}$. Suppose that $K$ is such a subalgebra with $\operatorname{dim}_{F} L / K=n$. Let $\mathscr{I}=\{0,1\}^{n}$ be the set of all multi-indices of length $n$ and put $\tau=(1,1, \ldots, 1)$. We say that two such multi-indices $I, J \in \mathscr{I}$ are disjoint if there is no component in which both are nonzero. The weight of $I$, which is defined to be the sum of the components, will be denoted by $|I|$. Let $\mathscr{U}(L)$ and $\mathscr{U}(K)$ be the universal enveloping algebras of $L$ and $K$, respectively, and choose $x_{1}, \ldots, x_{n}$ to be elements of $L_{1}$ whose cosets form a basis for $L / K$. For $I=(i(1), \ldots, i(n)) \in \mathscr{I}$, we define $\mathbf{x}^{I}=x_{1}^{i(1)} \cdots x_{n}^{i(n)}$. The Poincaré-Birkhoff-Witt Theorem ensures that every element of $\mathscr{U}(L)$ can be written uniquely in the form $u=\sum_{I \in \mathscr{I}} u_{I} \mathbf{x}^{I}$, with each $u_{I}$ lying in $\mathscr{U}(K)$.

We filter the $\mathscr{U}(K)$-module $\mathscr{U}(L)$ by setting $V(m):=\sum_{i=0}^{m} \mathscr{U}(K) \cdot\left(L_{1}^{i}\right)$ (so $V(0)=\mathscr{U}(K)$ and $V(n)=\mathscr{U}(L))$. A standard straightening argument shows that $V(m) \cdot \mathscr{U}(K) \subset V(m)$, qualifying $V(m)$ as a $\mathscr{U}(K)$-subbimodule of $\mathscr{U}(L)$. By applying the Poincaré-Birkhoff-Witt Theorem we thus obtain $V(m)=\bigoplus_{|I| \leq m} \mathscr{U}(K) \mathbf{x}^{I}=\bigoplus_{|I| \leq m} \mathbf{x}^{I} \mathscr{U}(K)$. Clearly, $V(k) V(l) \subseteq V(k+l)$ for all $k, l \geq 0$.

Lemma 2.1. (1) For $m \geq 2$, the product of any $m$ linearly dependent elements of $L_{1}$ lies in $V(m-2)$.

(2) Let $y_{1}, \ldots, y_{m} \in L_{1}$ and let $\sigma$ be a permutation in $S_{m}$. Then

$$
y_{\sigma(1)} \cdots y_{\sigma(m)}-(\operatorname{sgn} \sigma) y_{1} \cdots y_{m} \in V(m-2) \text {. }
$$

(3) If $I, J \in \mathscr{I}$, then one of $\mathbf{x}^{I} \mathbf{x}^{J}+\mathbf{x}^{I+J}$ or $\mathbf{x}^{I} \mathbf{x}^{J}-\mathbf{x}^{I+J}$ lies in $V(|I|+|J|-2)$.

Proof. (1) We may write the factors in the product as a linear combination of some subset of them, and so for the first claim it is enough to prove $y_{1} \cdots y_{m-1} y_{i}$ $\in V(m-2)$ whenever $y_{1}, \ldots, y_{m-1} \in L_{1}, 1 \leq i \leq m-1$. If $i=m-1$, then, observing $\left[y_{m-1}, y_{m-1}\right] \in L_{0}$, we obtain

$$
y_{1} \cdots y_{m-2} y_{m-1} y_{m-1}=\frac{1}{2} y_{1} \cdots y_{m-2}\left[y_{m-1}, y_{m-1}\right] \in V(m-2) \text {. }
$$

If $i<m-1$, then

$$
y_{1} \cdots y_{m-2} y_{m-1} y_{i}=-y_{1} \cdots y_{m-2} y_{i} y_{m-1}+y_{1} \cdots y_{m-2}\left[y_{m-1}, y_{i}\right] \text {, }
$$

with the second summand obviously being contained in $V(m-2)$, since $\left[y_{m-1}\right.$, $\left.y_{i}\right] \in L_{0}$. By induction on $m$ we may assume $y_{1} \cdots y_{m-2} y_{i} \in V(m-3)$, whence the element $y_{1} \cdots y_{m-2} y_{i} y_{m-1}$ belongs to $V(m-2)$.

(2), (3) To prove (2), we may assume without loss of generality that $\sigma$ is a transposition of the form $(i i+1)$. In this case

$$
\begin{aligned}
& y_{1} \cdots y_{i+1} y_{i} \cdots y_{m}+y_{1} \cdots y_{i} y_{i+1} \cdots y_{m} \\
& \quad=y_{1} \cdots y_{i-1}\left[y_{i}, y_{i+1}\right] y_{i+1} \cdots y_{m} \in V(m-2) .
\end{aligned}
$$

The definition of a Lie superalgebra implies that the map $f: K \rightarrow \operatorname{gl}(L / K)$ defined by $f(a)(y+K):=[a, y]+K$ for $a \in K, y \in L$ is a homomorphism of Lie superalgebras, and so $\lambda: K \rightarrow F$ defined by $\lambda(a):=\operatorname{tr}(f(a))=-\operatorname{str}(f(a))$ 
(where str stands for the supertrace) is a linear functional on $K$ which vanishes on $[K, K]+K_{1}$. Hence there is a unique automorphism $\alpha$ of $\mathscr{U}(K)$ such that

$$
\alpha(a)= \begin{cases}a+\lambda(a) 1 & \text { for } a \in K_{0}, \\ (-1)^{n} a & \text { for } a \in K_{1} .\end{cases}
$$

Theorem 2.2. Let $L$ be a Lie superalgebra, and $K \subset L$ a subalgebra of $L$ of codimension $n$ that contains $L_{0}$. Then the extension $\mathscr{U}(L): \mathscr{U}(K)$ is a free $\alpha$-Frobenius extension, where $\alpha$ is defined by $(*)$.

Proof. We will use the notation in the discussion preceding the theorem. We first consider the case $K=L_{0}$. Accordingly, let $x_{1}, \ldots, x_{n}$ be a basis for $L_{1}$. We define $\sigma: \mathscr{U}(L) \rightarrow \mathscr{U}\left(L_{0}\right)$ by $\sigma\left(\sum_{I \in \mathcal{I}} u_{I} \mathbf{x}^{I}\right)=u_{\tau}$ and consider the associated biadditive map $\langle\rangle:, \mathscr{U}(L) \times \mathscr{U}(L) \rightarrow \mathscr{U}\left(L_{0}\right) ;\langle u, v\rangle:=\sigma(u v)$. The Poincaré-Birkhoff-Witt Theorem shows that $\mathscr{U}(L)$ is a finitely generated free right and left $\mathscr{U}\left(L_{0}\right)$-module, each on the basis $\left\{\mathbf{x}^{I}: I \in \mathscr{I}\right\}$. The form $\langle$,$\rangle is evidently associative, and it is clear that if a$ is an element of $\mathscr{U}\left(L_{0}\right)$, then $\sigma(a u)=a \sigma(u)$, so $\langle a u, v\rangle=a\langle u, v\rangle$. Defining $\beta: \mathscr{U}\left(L_{0}\right) \rightarrow \mathscr{U}\left(L_{0}\right)$ via $\beta(x)=x+\operatorname{tr}\left(\left.\operatorname{ad} x\right|_{L_{1}}\right)$ for $x \in L_{0}$, we claim $\sigma(u a)=\sigma(u) \beta^{-1}(a)$ for all $u \in \mathscr{U}(L), a \in \mathscr{U}\left(L_{0}\right)$.

The Poincaré-Birkhoff-Witt Theorem shows that the multiplication map of $\mathscr{U}(L)$ induces an isomorphism

$$
\mathscr{U}\left(L_{0}\right) \otimes_{F} \Lambda\left(L_{1}\right) \cong \mathscr{U}(L)
$$

of $L_{0}$-modules, with $L_{0}$ acting on $\mathscr{U}\left(L_{0}\right)$ and the exterior algebra $\Lambda\left(L_{1}\right)$ via the adjoint representation. Recall that $\Lambda\left(L_{1}\right)$ is a graded $L_{0}$-module on whose $n$th component $L_{0}$ operates via the map $x \mapsto \operatorname{tr}\left(\left.\operatorname{ad} x\right|_{L_{1}}\right)$. Now let $a \in L_{0}$ and $u \in \mathscr{U}\left(L_{0}\right)$. Then we have

$$
\begin{aligned}
\sigma\left(\left[a, u \mathbf{x}^{I}\right]\right) & =\sigma\left([a, u] \mathbf{x}^{I}\right)+\sigma\left(u\left[a, \mathbf{x}^{I}\right]\right)=\delta_{I, \tau}[a, u]+\delta_{I, \tau} \lambda(a) u \\
& =\left[a, \sigma\left(u \mathbf{x}^{I}\right)\right]+\lambda(a) \sigma\left(u \mathbf{x}^{I}\right) .
\end{aligned}
$$

Consequently,

$$
\sigma([a, r])=[a, \sigma(r)]+\lambda(a) \sigma(r) \quad \text { for all } a \in L_{0}, \quad r \in \mathscr{U}(L) .
$$

This implies

$$
\sigma(r a)=\sigma(a r-[a, r])=a \sigma(r)-[a, \sigma(r)]-\lambda(a) \sigma(r)=\sigma(r) \beta^{-1}(a)
$$

for every $a \in L_{0}$ and $r \in \mathscr{U}(L)$. Thus $\sigma(r s)=\sigma(r) \beta^{-1}(s)$ for every $s \in \mathscr{U}\left(L_{0}\right)$ and $r \in \mathscr{U}(L)$.

Next define any total order $\ll$ on $\mathscr{I}$ which has the property that $I \ll J$ implies $|I| \leq|J|$. Let $I^{\prime}$ denote the complement $\tau-I$ of $I$, so $\left|I^{\prime}\right|=n-|I|$. If $|I|<|J|$, then $|I|+\left|J^{\prime}\right|<n$, so $\mathbf{x}^{I} \mathbf{x}^{J^{\prime}} \in V(n-1)$. If $|I|=|J|$ and $I \neq J$, then $|I|+\left|J^{\prime}\right|=n$, but $I$ and $J^{\prime}$ are not disjoint (i.e., $\mathbf{x}^{I}$ and $\mathbf{x}^{J^{\prime}}$ have some variables in common), so (2.1)(1) yields $\mathbf{x}^{I} \mathbf{x}^{J^{\prime}} \in V(n-2)$. This shows that if $I \ll J$ and $I \neq J$, then $\left\langle\mathbf{x}^{I}, \mathbf{x}^{J^{\prime}}\right\rangle=0$. Applying (2.1) again we see that $\left\langle\mathbf{x}^{I}, \mathbf{x}^{I^{\prime}}\right\rangle= \pm 1$.

For $I \in \mathscr{I}$ put $x_{I}:=\mathbf{x}^{I}$ and $y_{I}:=\mathbf{x}^{I^{\prime}}$. Then $\left\{x_{I}: I \in \mathscr{I}\right\}$ and $\left\{y_{I}: I \in\right.$ $\mathscr{I}\}$ are ordered bases of the left and right $\mathscr{U}\left(L_{0}\right)$-module $\mathscr{U}(L)$, respectively. Consider the matrix $\left[\left\langle x_{I}, y_{J}\right\rangle\right]_{I, J \in \mathcal{I}}$. According to the last paragraph this is 
a lower triangular matrix whose diagonal entries are all \pm 1 . Thus by $(1.2)(\mathrm{c})$, $\mathscr{U}(L)$ is a free $\beta$-Frobenius extension of $\mathscr{U}\left(L_{0}\right)$.

Now suppose that $K$ is as given in the theorem. Let $x_{1}, \ldots, x_{n}$ be elements of $L_{1}$ whose cosets form a basis of $L / K$ and let $w_{1}, \ldots, w_{m}$ be a basis for $K_{1}$. Adopting the notation introduced above, we define $\pi: \mathscr{U}(L) \rightarrow \mathscr{U}(K)$ by $\pi\left(\sum_{I \in \mathcal{I}} u_{I} \mathbf{x}^{I}\right):=u_{\tau}$, where the coefficients $u_{I}$ are in $\mathscr{U}(K)$. By the first part of the proof, $\mathscr{U}(L): \mathscr{U}\left(L_{0}\right)$ is a $\beta$-Frobenius extension, with the Frobenius homomorphism $\sigma$ being the projection of an element of $\mathscr{U}(L)$ onto the coefficient (from $\mathscr{U}\left(L_{0}\right)$ ) of the monomial $w_{1} \cdots w_{m} x_{1} \cdots x_{n}$. Likewise, $\mathscr{U}(K): \mathscr{U}\left(L_{0}\right)$ is a $\gamma$-Frobenius extension, where $\gamma(x)=x+\operatorname{tr}\left(\left.\operatorname{ad} x\right|_{K_{1}}\right)$ for $x \in L_{0}$, with the Frobenius homomorphism $\rho$ being the projection of an element of $\mathscr{U}(K)$ onto the coefficient (from $\mathscr{U}\left(L_{0}\right)$ ) of the monomial $w_{1} \cdots w_{m}$. Obviously $\rho \circ \pi=\sigma$, and we have noted before that $\mathscr{U}(L)$ is free over $\mathscr{U}(K)$. By (1.4), to show $\mathscr{U}(L): \mathscr{U}(K)$ is an $\alpha$-Frobenius extension with Frobenius homomorphism $\pi$, it suffices to verify

$$
\pi(u a)=\pi(u) \alpha^{-1}(a) \text { for all } u \in \mathscr{U}(L), a \in K .
$$

By definition we have $\alpha(a)=\beta \circ \gamma^{-1}(a)$ for $a \in \mathscr{U}\left(L_{0}\right)$, so it is enough to show this equality for $a \in K_{1}$.

Let $a \in K_{1}$ and let $u \in \mathscr{U}(K)$. For $I \in \mathscr{I}$, we obtain as a consequence of (2.1) that $u \mathbf{x}^{I} a \equiv(-1)^{n} u a \mathbf{x}^{I}(\bmod V(n-1))$, so that

$$
\pi\left(u \mathbf{x}^{I} a\right)=(-1)^{n} u a \delta_{I, \tau}=\pi\left(u \mathbf{x}^{I}\right) \alpha^{-1}(a) .
$$

It now follows that $\pi(r b)=\pi(r) \alpha^{-1}(b)$ for all $r \in \mathscr{U}(L)$ and all $b \in \mathscr{U}(K)$.

Throughout the remainder of this section $L=L_{0} \oplus L_{1}$ is assumed to be a finite-dimensional, $\mathbb{Z}$-graded Lie superalgebra, i.e., $L=\bigoplus_{i \in \mathbb{Z}} L_{(i)}$ with each $L_{(i)}$ a homogeneous subspace and with $\left[L_{(i)}, L_{(j)}\right] \subset L_{(i+j)}$. We consider $L^{-}:=$ $\bigoplus_{i<0} L_{(i)}$ and $L^{+}:=\bigoplus_{i>0} L_{(i)}$ and define $\mathscr{P}:=L_{(0)} \oplus L^{+}$. Using the PoincaréBirkhoff-Witt Theorem one readily verifies the following facts for the $\mathbb{Z}$-grading inherited by $\mathscr{U}(L)$ :

(i) $\mathscr{U}(\mathscr{P})=\bigoplus_{i \geq 0} \mathscr{U}(\mathscr{P})_{(i)} ; \mathscr{U}(\mathscr{P})_{(i)} \subset \mathscr{U}\left(L_{(0)}\right) \mathscr{U}\left(L^{+}\right)^{+}$for all $i>0$, where $\mathscr{U}\left(L^{+}\right)^{+}$is the augmentation ideal of $\mathscr{U}\left(L^{+}\right)$(that is, the kernel of the canonical supplementation on $\left.\mathscr{U}\left(L^{+}\right)\right)$.

(ii) $\mathscr{U}\left(L^{-}\right)=\bigoplus_{i \leq 0} \mathscr{U}\left(L^{-}\right)_{(i)} ; \mathscr{U}\left(L^{-}\right)_{(0)}=F \cdot 1$.

Following Shen [16], we refer to a $\mathbb{Z}$-graded $L$-module $\mathscr{V}$ as positively graded if $\mathscr{V}=\bigoplus_{i \geq 0} \mathscr{V}_{(i)}$. A positively graded module $\mathscr{V}$ is said to be transitively graded if $\mathscr{V}_{(0)}=\left\{v \in \mathscr{V}: x \cdot v=0\right.$ for all $\left.x \in L^{-}\right\}$.

Let $V$ be an $L_{(0)}$-module. We extend the operation on $V$ to $\mathscr{P}$ by letting $L^{+}$act trivially. Note that the augmentation ideal $\mathscr{U}\left(L^{+}\right)^{+}$of $\mathscr{U}\left(L^{+}\right)$then annihilates $V$. We shall be studying the coinduced module $\operatorname{Coind}_{\mathscr{P}}(V):=$ $\operatorname{Hom}_{\mathscr{U}(\mathscr{P})}(\mathscr{U}(L), V)$.

Theorem 2.3. Let $V$ be an $L_{(0)}$-module and suppose $L_{0} \subset \mathscr{P}$.

(1) The L-module $\mathscr{V}:=$ Coind $_{\mathscr{P}}(V)$ obtains the structure of a transitively graded L-module by setting $\mathscr{V}_{(i)}:=\left\{f \in \mathscr{V}: f\left(\mathscr{U}(L)_{(j)}\right)=(0)\right.$ for all $\left.j \neq-i\right\}$.

(2) $\mathscr{V}_{(0)}$ is isomorphic to $V$ as an $L_{(0)}$-module.

Suppose, in addition, that $V$ is finite dimensional.

(3) If $V$ is indecomposable, then $\mathscr{V}$ is indecomposable. 
(4) If $V$ is irreducible, $V=\mathscr{U}\left(L_{(0)}\right) v_{0}$, then $\mathscr{V}$ contains a unique irreducible submodule, which is generated by the image of $v_{0}$ under the isomorphism in (2). Proof. (1) Let $f$ be an element of $\mathscr{V}_{(i)}, u \in \mathscr{U}(L)_{(k)}$, and suppose that $j \neq$ $-i-k$. If $x \in \mathscr{U}(L)_{(j)}$, then $x u \in \mathscr{U}(L)_{(j+k)}$ and as $j+k \neq-i$ we obtain $(u \cdot f)(x)=f(x u)=0$. Consequently, $(u \cdot f)$ belongs to $\mathscr{V}_{(i+k)}$.

Now let $\left\{u_{1}, \ldots, u_{t}\right\}$ be the basis of $\mathscr{U}(L)$ over $\mathscr{U}(\mathscr{P})$ induced by a basis $\left\{e_{1}, \ldots, e_{k}\right\}$ of $L^{-}$. (Such a basis is finite since $L_{0} \subset \mathscr{P}$.) Owing to (ii) we have $u_{l} \in \mathscr{U}(L)_{(i(l))}$, where $i(l) \leq 0$ for $1 \leq l \leq t$. For an arbitrary element $v \in V$ and $l \in\{1, \ldots, t\}$, let $X_{v}^{(l)}: \mathscr{U}(L) \rightarrow V$ be the $\mathscr{U}(\mathscr{P})$-module homomorphism given by $X_{v}^{(l)}\left(u_{i}\right):=\delta_{l i} v$ for $1 \leq i \leq t$. Any element $u \in$ $\mathscr{U}(L)_{(k)}$ has a representation $u=\sum_{l=1}^{t} r_{l} u_{l}$, where $r_{l} \in \mathscr{U}(\mathscr{P})_{(k-i(l))}$ for $1 \leq$ $l \leq t$. Given $l \in\{1, \ldots, t\}$, we have $X_{v}^{(l)}(u)=r_{l} v$. If $k \neq i(l)$, then (i) in conjunction with the definition of the $\mathscr{U}(\mathscr{P})$-module structure of $V$ forces the vanishing of $X_{v}^{(l)}(u)$. This qualifies $X_{v}^{(l)}$ as an element of $\mathscr{V}_{(-i(l))}$. For every element $f \in \mathscr{V}$ we have $f=\sum_{l=1}^{t} X_{f\left(u_{l}\right)}^{(l)}$. Consequently, $\mathscr{V}=\bigoplus_{l=1}^{t} \mathscr{V}_{(-i(l))}$, proving that $\mathscr{V}$ is a positively graded module.

To verify the transitivity of $\mathscr{V}$, let $f$ be an element of $\mathscr{V}$ such that $x \cdot f=0$ for every $x \in L^{-}$. Since each homogeneous constituent of $f$ enjoys the same property, we may assume $f$ to be homogeneous of degree $l$. Suppose that $l>0$ : we may write any element of $\mathscr{U}(L)_{(-l)}$ as a sum of elements of the form $u=\sum_{i+k=-l} a_{i} b_{k}$, where $a_{i} \in \mathscr{U}(\mathscr{P})_{(i)}$ and $b_{k} \in \mathscr{U}\left(L^{-}\right)_{(k)}$. Owing to (i) we have $a_{i} \cdot V=(0)$ for $i>0$, and so

$$
f(u)=\sum_{i+k=-l} a_{i} f\left(b_{k}\right)=a_{0} f\left(b_{-l}\right)=a_{0}\left(b_{-l} \cdot f\right)(1) .
$$

By virtue of (ii), $b_{-l}$ is contained in $\mathscr{U}\left(L^{-}\right)^{+}$and therefore annihilates $f$. Thus $f(u)=0$ and it follows that $f$ is the zero map. This shows $\mathscr{V}$ is transitively graded.

(2) We proceed by showing that $\lambda: \mathscr{V}_{(0)} \rightarrow V ; \lambda(f):=f(1)$ is an isomorphism of $L_{(0)}$-modules. If $x \in L_{(0)}$, then

$$
\lambda(x \cdot f)=(x \cdot f)(1)=f(x)=x f(1)=x \lambda(f),
$$

and so $\lambda$ is a homomorphism of $L_{(0)}$-modules. Since $1=u_{l_{0}} \in \mathscr{U}(L)_{(0)}$ is, according to definition, contained in $\left\{u_{1}, \ldots, u_{t}\right\}$, the corresponding map $X_{v}^{\left(l_{0}\right)}$ is a preimage of $v \in V$ under $\lambda$. Suppose that $f \in \operatorname{ker} \lambda$. Owing to the Poincaré-Birkhoff-Witt Theorem, every element of $\mathscr{U}(L)_{(0)}$ is a sum of elements of the form $u=\sum_{i+k=0} a_{i} b_{k}$, where $a_{i} \in \mathscr{U}(\mathscr{P})_{(i)}$ and $b_{k} \in \mathscr{U}\left(L^{-}\right)_{(k)}$. Since, according to (i), $a_{i}=0$ for $i<0$ and $a_{i} \in \mathscr{U}\left(L_{(0)}\right) \mathscr{U}\left(L^{+}\right)^{+}$for $i>0$, we obtain, observing (ii),

$$
f(u)=\sum_{i+k=0} a_{i} f\left(b_{k}\right)=a_{0} f\left(b_{0}\right)=a_{0} b_{0} f(1)=0 .
$$

As a result, $f$ vanishes on $\mathscr{U}(L)_{(0)}$ and thereby on all of $\mathscr{U}(L)$.

(3), (4) Assume that $V$ is finite dimensional. As $\mathscr{U}(L)$ is a free module of finite rank over $\mathscr{U}(\mathscr{P}), \mathscr{V}$ is finite dimensional. Let $U \subset \mathscr{V}$ be any nonzero submodule. The associative $F$-algebra $\mathscr{U}\left(L^{-}\right)^{+}$obviously acts on $\mathscr{V}$ 
by nilpotent transformations. The Engel-Jacobson Theorem (cf. [7, p. 33]) thus implies that $U_{0}\left(L^{-}\right):=\left\{v \in U: x \cdot v=0\right.$ for all $\left.x \in L^{-}\right\}$is not trivial.

If $\mathscr{V}=U \oplus W$ is a decomposition of $\mathscr{V},(1)$ ensures that $\mathscr{V}_{(0)}=U_{0}\left(L^{-}\right) \oplus$ $W_{0}\left(L^{-}\right)$is a decomposition of $\mathscr{V}_{(0)}$ into $L_{(0)}$-modules. It now follows from (2) and our present assumption that $U_{0}\left(L^{-}\right)=(0)$ or $W_{0}\left(L^{-}\right)=(0)$. Hence the initially given decomposition of $\mathscr{V}$ is trivial, by the observation in the last paragraph.

In case $V$ is irreducible and $U \neq(0)$ is an $L$-submodule of $\mathscr{V}$, we obtain from (2) that $U_{0}\left(L^{-}\right)$coincides with $\mathscr{V}_{(0)}$. Thus the submodule generated by $\lambda^{-1}\left(v_{0}\right)$ is contained in $U$ and hence is the unique irreducible submodule of $\mathscr{V}$.

We now specialize our considerations to basic classical Lie superalgebras over an algebraically closed field of characteristic 0 . According to [9] such a Lie superalgebra is simple with a reductive Lie algebra as subalgebra of even elements. Let $H$ be a Cartan subalgebra of $L_{0}$. Then there exists a triangular decomposition $L=N^{-} \oplus H \oplus N^{+}$of subalgebras of $L$. An $L$-module $V$ is said to be a highest weight module of $L$ with highest weight $\lambda \in H^{*}$, if there is $v_{\lambda} \in V$ such that

(a) $V=\mathscr{U}(L) v_{\lambda}$,

(b) $h \cdot v_{\lambda}=\lambda(h) v_{\lambda}$ for every $h \in H$, and

(c) $N_{0}^{+} \cdot v_{\lambda}=(0)$.

If $L$ is of type $\mathrm{I}$, it admits a so-called distinguished $\mathbb{Z}$-gradation of the form $L=L_{(-1)} \oplus L_{(0)} \oplus L_{(1)}$, for which $\mathscr{P}=L_{0}+N^{+}=L_{(0)} \oplus L_{(1)}$ and $N_{1}^{-}=L_{(-1)}$. If $\rho_{1}$ denotes the half sum of all odd positive roots, then $\operatorname{tr}\left(\left.\operatorname{ad} h\right|_{N_{1}^{-}}\right)=-2 \rho_{1}(h)$ for all $h \in H$. Given an irreducible highest weight module $V(\lambda)$ for $L_{0}$, Kac [9] studied the induced modules $\operatorname{Ind}_{\mathscr{P}}(V(\lambda)):=\mathscr{U}(L) \otimes_{\mathscr{U}(\mathscr{P})} V(\lambda)$. Note that Ind $_{\mathscr{P}}(V(\lambda))$ is a highest weight module of $L$ with highest weight $\lambda$ and highest weight vector $1 \otimes v_{\lambda}$.

Corollary 2.4. Let $L=L_{0} \oplus L_{1}$ be a basic classical Lie superalgebra of type I and $V(\lambda)$ a finite-dimensional irreducible highest weight module for $L_{0}$. Then Ind $_{\mathscr{P}}(V(\lambda))$ contains a unique irreducible submodule, which is of highest weight $\lambda-2 \rho_{1}$.

Proof. Since $L_{0}$ is contained in $\mathscr{P}, \mathscr{U}(L): \mathscr{U}(\mathscr{P})$ is, in accordance with (2.2), an $\alpha$-Frobenius extension. In view of the isomorphism

$$
\operatorname{Ind}_{\mathscr{P}}(V(\lambda)) \cong \operatorname{Coind}_{\mathscr{P}}\left({ }_{\alpha} V(\lambda)\right)
$$

(cf. §3) it suffices to establish the assertion for the coinduced module. Since $N_{0}^{+}$operates nilpotently on $L$, we have $\alpha(x)=x$ for every $x \in N_{0}^{+}$. Hence the highest weight vector $v_{\lambda}$ of the $L_{0}$-module $V(\lambda)$ is a highest weight vector of highest weight $\lambda-2 \rho_{1}$ for the irreducible $L_{0}$-module ${ }_{\alpha} V(\lambda)$. Since $L_{0}$ is contained in $\mathscr{P}$ and $V(\lambda)$ is finite dimensional, (2.3)(4) applies and the coinduced module Coind ${ }_{\mathscr{P}}\left({ }_{\alpha} V(\lambda)\right)$ contains a unique irreducible submodule, which is generated by a highest weight vector of highest weight $\lambda-2 \rho_{1}$.

\section{Cohomological observations}

Suppose $R: S$ is an $\alpha$-Frobenius extension, and let $V$ be an $R$-module. The theory of Frobenius extensions[13, p. 96f] provides a natural equivalence 


$$
R \otimes_{S} V \cong \operatorname{Hom}_{S}\left(R,{ }_{\alpha} V\right) .
$$

Consequently, there exists a projection $\operatorname{Hom}_{S}\left(R,{ }_{\alpha} N\right) \rightarrow N$ for every $R$ module $N$. By Frobenius reciprocity there result corestriction maps

$$
\operatorname{Cor}_{[R: S]}^{n}: \operatorname{Ext}_{S}^{n}\left(M,{ }_{\alpha} N\right) \rightarrow \operatorname{Ext}_{R}^{n}(M, N)
$$

for any two $R$-modules $M$ and $N$ (cf. [2, §3]). Note that by [2, 3.1(1)], $\operatorname{Cor}_{[R: S]}^{0}=\operatorname{Tr}_{[R: S]}$, so the following result generalizes part of (1.3).

Theorem 3.1. Let $T \subset S \subset R$ be rings such that

(a) $R: S$ is an $\alpha$-Frobenius extension,

(b) $S: T$ is a $\gamma$-Frobenius extension, and

(c) $\alpha(T)=T$.

If $M$ and $N$ are $R$-modules, then $\operatorname{Cor}_{[R: T]}^{n}=\operatorname{Cor}_{[R: S]}^{n}{ }^{\circ} \operatorname{Cor}_{[S: T]}^{n}$ for all $n \geq 0$. Proof. We shall proceed by induction on $n$, the case $n=0$ following from (1.3). Now suppose that $n \geq 1$. Let $(0) \rightarrow X \rightarrow P \rightarrow M \rightarrow(0)$ be a projective presentation of the $R$-module $M$. Since the rings involved are projective over the relevant subrings, the above sequence is also a projective presentation of $M$ viewed as an $S$ - and $T$-module, respectively. The corestriction mappings are natural transformations that commute with connecting homomorphisms. The long exact cohomology sequence thus yields the following commutative diagram:

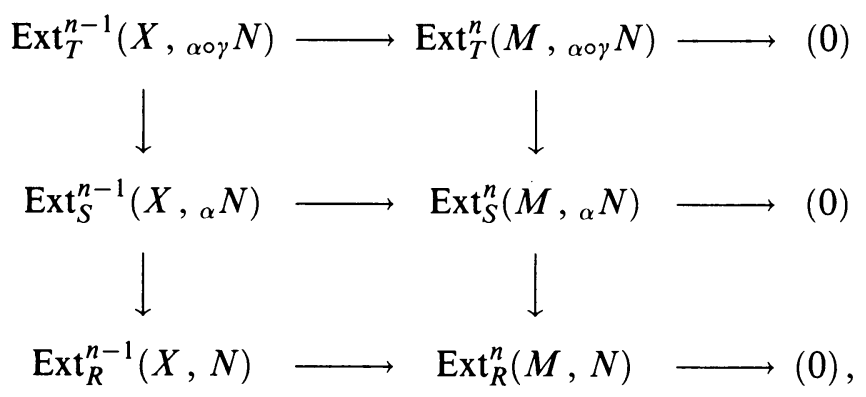

where the vertical arrows represent the relevant corestriction mappings. By the inductive hypothesis our claim holds for the corestriction maps of level $n-1$. The assertion now follows from a standard argument.

We let $\operatorname{Res}_{[R: S]}^{n}: \operatorname{Ext}_{R}^{n}(M, N) \rightarrow \operatorname{Ext}_{S}^{n}(M, N)$ denote the natural change of rings map. The following result provides a generalization of [2, (3.1)].

Theorem 3.2. Let $R: S$ be an $\alpha$-Frobenius extension and $X, M, N$ be $R$ modules. Let $f$ be an element of $\operatorname{Hom}_{S}\left(M,{ }_{\alpha} N\right)$. Then the following statements hold:

(1) $\operatorname{Ext}_{R}^{n}\left(\operatorname{id}_{X}, \operatorname{Tr}_{[R: S]}(f)\right)=\operatorname{Cor}_{[R: S]}^{n} \circ \operatorname{Ext}_{S}^{n}\left(\operatorname{id}_{X}, f\right) \circ \operatorname{Res}_{[R: S]}^{n}$ for all $n \geq 0$. $\operatorname{Ext}_{R}^{n}\left(\operatorname{Tr}_{[R: S]}(f), \mathrm{id}_{X}\right)=\operatorname{Cor}_{[R: S]}^{n} \circ \operatorname{Ext}_{S}^{n}\left(f, \mathrm{id}_{X}\right) \circ \operatorname{Res}_{[R: S]}^{n}$ for all $n \geq 0$.

(2) Suppose that $\operatorname{Ext}_{R}^{n+1}\left(X, \operatorname{ker} \operatorname{Tr}_{[R: S]}(f)\right)=(0)$ for some $n \geq 0$ and $\operatorname{Tr}_{[R: S]}(f)$ is surjective.

(a) If $\operatorname{Ext}_{S}^{n}(X, M)=(0)$ or $\operatorname{Ext}_{S}^{n}\left(X,{ }_{\alpha} N\right)=(0)$, then $\operatorname{Ext}_{R}^{n}(X, N)=(0)$.

(b) If $\operatorname{Ext}_{S}^{n+1}(X, M)=(0)$ or $\operatorname{Ext}_{S}^{n+1}\left(X,{ }_{\alpha} N\right)=(0)$, then $\operatorname{Ext}_{R}^{n+1}(X, M)=$ $(0)$. 
Proof. (1) We proceed by induction on $n$. For $n=0$, both identities follow via direct computation using $\operatorname{Cor}_{[R: S]}^{0}=\operatorname{Tr}_{[R: S]}$. Since all maps involved are natural in $X$, the argument of the proof of (3.1) may now be adopted mutatis mutandis to yield our assertions.

(2)(a) By considering the long exact cohomology sequence induced by $(0) \rightarrow$ $\operatorname{ker} \operatorname{Tr}_{[R: S]}(f) \rightarrow M \rightarrow N \rightarrow(0)$ in conjunction with the hypothesis

$$
\operatorname{Ext}_{R}^{n+1}\left(X, \operatorname{ker} \operatorname{Tr}_{[R: S]}(f)\right)=(0),
$$

we obtain the surjectivity of the map $\operatorname{Ext}_{R}^{n}\left(\mathrm{id}_{X}, \operatorname{Tr}_{[R: S]}(f)\right): \operatorname{Ext}_{R}^{n}(X, M) \rightarrow$ $\operatorname{Ext}_{R}^{n}(X, N)$. If $\operatorname{Ext}_{S}^{n}(X, M)$ or $\operatorname{Ext}_{S}^{n}\left(X,{ }_{\alpha} N\right)$ is trivial, then $\operatorname{Ext}_{S}^{n}\left(\operatorname{id}_{X}, f\right)$ vanishes and the above factorization identifies $\operatorname{Ext}_{R}^{n}\left(\operatorname{id}_{X}, \operatorname{Tr}_{[R: S]}(f)\right)$ as the zero map. Consequently, $\operatorname{Ext}_{R}^{n}(X, N)=(0)$.

(b) In this situation $\operatorname{Ext}_{S}^{n+1}\left(\operatorname{id}_{X}, f\right)$ is trivial and $\operatorname{Ext}_{R}^{n+1}\left(\operatorname{id}_{X}, \operatorname{Tr}_{[R: S]}(f)\right)$ is injective. Consequently, $\operatorname{Ext}_{R}^{n+1}(X, M)=(0)$.

Remark. If $\alpha$ can be extended to an endomorphism of $R$, then the first part of the preceding result, applied to $\operatorname{id}_{N} \in \operatorname{Hom}_{S}\left({ }_{\alpha} N,{ }_{\alpha} N\right)$, readily yields [2, $(3.1)(2)]$.

Given an $R$-module $M$, we let inj. $\operatorname{dim}_{R} M$ denote its injective dimension.

Corollary 3.3. Let $R: S$ be an $\alpha$-Frobenius extension, $X$ and $M$ be $R$ modules, and suppose that $M$ is $(R, S)$-injective. Then the following statements hold:

(1) The restriction map $\operatorname{Res}_{[R: S]}^{n}: \operatorname{Ext}_{R}^{n}(X, M) \rightarrow \operatorname{Ext}_{S}^{n}(X, M)$ is injective.

(2) inj. $\operatorname{dim}_{R} M=\operatorname{inj} \cdot \operatorname{dim}_{S} M$. In particular, $M$ is $R$-injective if and only if it is $S$-injective.

Proof. (1) Owing to [15, Satz 11] there exists $f \in \operatorname{Hom}_{S}\left(M,{ }_{\alpha} M\right)$ such that $\operatorname{Tr}_{[R: S]}(f)=\mathrm{id}_{M}$. It thus follows from (3.2)(1) that $\operatorname{Cor}_{[R: S]}^{n} \circ \operatorname{Ext}_{S}^{n}\left(\operatorname{id}_{X}, f\right) \circ$ $\operatorname{Res}_{[R: S]}^{n}$ is the identity operator on $\operatorname{Ext}_{R}^{n}(X, M)$, thereby proving the injectivity of $\operatorname{Res}_{[R: S]}^{n}$.

(2) It suffices to prove that inj. $\operatorname{dim}_{R} M \leq \mathrm{inj} . \operatorname{dim}_{S} M$. This is a direct consequence of (1).

An analogous result holds for projective dimensions.

We shall next study the relation between the cohomology theories of $L$ and $L_{0}$ under the hypothesis that $L$ is finite dimensional. Our attention will be focused on the case where the Lie algebra $L_{0}$ is semireductive in the sense that it is the direct sum of a nilpotent and a semisimple ideal. Assuming the characteristic of the underlying base field to be zero, we then have $\operatorname{tr}\left(\operatorname{ad}_{L_{0}}(x)\right)=$ 0 for every $x \in L_{0}$. Consequently, the automorphism $\alpha$ of the Frobenius extension $\mathscr{U}(L): \mathscr{U}\left(L_{0}\right)$ is the restriction of the automorphism of $\mathscr{U}(L)$ that maps an element $x$ of $L$ onto $x-\operatorname{str}\left(\operatorname{ad}_{L}(x)\right) 1$.

In general, consider a subalgebra $K$ of $L$ that contains $L_{0}$. If the automorphism $\alpha$ of $\mathscr{U}(K)$ can be extended to an endomorphism $\alpha$ of $\mathscr{U}(L)$ (this is not always the case) and $\left\{x_{1}, \ldots, x_{m}\right\},\left\{y_{1}, \ldots, y_{m}\right\}$ is a dual free pair for the Frobenius extension $\mathscr{U}(L): \mathscr{U}(K)$, we let $c_{0}:=\sum_{i=1}^{m} y_{i} \alpha\left(x_{i}\right)$ denote the Casimir element of the extension (cf. [2]). This element has the property that $r c_{0}=c_{0} \alpha(r)$ for all $r \in \mathscr{U}(L)$.

Given an $L$-module $M$, we shall consider $H^{n}(L, M):=\operatorname{Ext}_{\mathscr{U}(L)}^{n}(F, M)$, 
the cohomology groups of $L$ with coefficients in $M$. Let $\varepsilon: \mathscr{U}(L) \rightarrow F$ be the canonical supplementation.

Proposition 3.4. Suppose that $\operatorname{char}(F)=0$ and let $L_{0}$ be semireductive. Given a finite-dimensional $L$-module $M$, define $M^{L_{0}}:=\{m \in M \mid x \cdot m=0$ for all $\left.x \in L_{0}\right\}$. Then the following statements hold:

(1) Suppose that $M^{L_{0}}=\{0\}$. If there exists $f \in \operatorname{Hom}_{\mathscr{U}\left(L_{0}\right)}\left(M,{ }_{\alpha} M\right)$ such that $\operatorname{Tr}(f)$ is a bijection, then $H^{n}(L, M)=(0)$ for all $n \geq 0$.

(2) Suppose that $M^{L_{0}}=\{0\}$. If $\varepsilon\left(c_{0}\right) \neq 0$, then $H^{n}(L, M)=(0)$ for all $n \geq 0$.

(3) If $L_{0}$ is semisimple and $c_{0}$ operates invertibly on $M$, then $M$ is completely reducible.

(4) If $L_{0}$ is semisimple and if either $c_{0}$ operates invertibly on $M$ or $\varepsilon\left(c_{0}\right) \neq 0$, then $H^{n}(L, M)=(0)$ for $1 \leq n \leq 2$.

Proof. (1) We apply (3.2) to the case $X:=F$ and $N:=M$. Since $\operatorname{ker} \operatorname{Tr}(f)=$ $(0)$, we have that $\operatorname{Ext}_{\mathscr{U}(L)}^{n}(F, \operatorname{ker} \operatorname{Tr}(f))$ vanishes for all $n$. On the other hand, $[1,(3.1)]$ shows that $H^{n}\left(L_{0}, M\right)=(0)$ for $n \geq 0$. Consequently, (3.2) gives the desired result.

(2) According to [2, (3.1), (3.2); 1, (3.1)], the map $\operatorname{Cor}_{n} \circ \operatorname{Res}_{n}$ is invertible while $\operatorname{Res}_{n}$ is the zero map. Consequently, $H^{n}(L, M)$ is trivial for all $n$.

(3) Let $V \subset M$ be a submodule. By the first Whitehead Lemma, we have $\operatorname{Ext}_{\mathscr{U}\left(L_{0}\right)}^{1}(M / V, V) \cong H^{1}\left(L_{0}, \operatorname{Hom}_{F}(M / V, V)\right)=(0)$. Since $c_{0}$ operates invertibly on $V$, the map $\operatorname{Cor}_{n} \circ \operatorname{Res}_{n}$ is invertible, and we obtain the vanishing of $\operatorname{Ext}_{\mathscr{U}(L)}^{1}(M / V, V)$. Hence $V$ is a direct summand of $M$.

(4) The proof is analogous to (2) with the exception that the Whitehead Lemmas (cf. [7, pp. 77, 89]) are used in lieu of [1, (3.1)].

Remarks. (1) If $\alpha=\operatorname{id}_{\mathscr{U}\left(L_{0}\right)}$, then $c_{0}$ lies in the center of $\mathscr{U}(L)$, and any finite-dimensional $L$-module $M$ admits a decomposition $M=M_{0} \oplus M_{1}$ into $L$-stable Fitting components relative to $c_{0}$. By the preceding result, $M_{1}$ is a completely reducible $L$-module.

(2) Suppose that $L$ is a basic classical Lie superalgebra of type I. Let Ind $_{\mathscr{P}}(V(\lambda))$ be the highest weight module defined by an irreducible $L_{0}$-module $V(\lambda)$. Then $\operatorname{Ind}_{\mathscr{P}}(V(\lambda))$ is indecomposable and the Casimir element operates on Ind $\mathscr{P}(V(\lambda))$ by a scalar $\lambda_{0}$. If $\lambda_{0}$ is not zero, then the above observations ensure the irreducibility of $\operatorname{Ind}_{\mathscr{P}}(V(\lambda))$.

Example. The Lie superalgebra $\operatorname{osp}(1,2)$ is the set of matrices of the form

$$
\left[\begin{array}{c|cc}
0 & d & -c \\
\hline c & r & s \\
d & t & -r
\end{array}\right]
$$

with the usual supercommutator; note that $L_{0} \cong \operatorname{sl}(2)$. A basis for $L_{1}$ is given by $x=e_{21}-e_{13}, y=e_{31}+e_{12}$. Thus the left $\mathscr{U}\left(L_{0}\right)$-module $\mathscr{U}(L)$ has a basis consisting of $x_{1}=1, x_{2}=x, x_{3}=y, x_{4}=x y+1-[x, y]$. A dual basis with respect to $\langle$,$\rangle for \mathscr{U}(L)$ as a right $\mathscr{U}\left(L_{0}\right)$-module is given by $y_{1}=x y, y_{2}=y, y_{3}=-x, y_{4}=1$. Now

$$
c_{0}=\sum_{i=1}^{4} y_{i} x_{i}=x y+y x-x y+x y+1-[x, y]=1 \text {. }
$$


Thus (3.4)(3) applies and every finite-dimensional osp(1,2)-module is completely reducible. This fact was first observed by Hochschild in [6].

Corollary 3.5. Suppose that $L=L_{0} \oplus L_{1}$ is a Lie superalgebra with semireductive even part over a field $F$ of characteristic 0 . Let $K$ be a subalgebra of $L$ containing $L_{0}$ and $V$ be a finite-dimensional $K$-module. Suppose that

(a) $\left\{v \in V: x \cdot v=-\operatorname{tr}\left(\operatorname{ad}_{L / K}(x)\right) v\right.$ for all $\left.x \in L_{0}\right\}=(0)$, and

(b) the Casimir element $c_{0}$ of the extension $\mathscr{U}(K): \mathscr{U}\left(L_{0}\right)$ operates nontrivially on $F$.

Then $H^{n}\left(L, \operatorname{Ind}_{K}(V)\right)=(0)$ for all $n \geq 0$.

Proof. Let $\alpha$ be the automorphism of the Frobenius extension $\mathscr{U}(L): \mathscr{U}(K)$. By Frobenius reciprocity in conjunction with the natural equivalence

$$
\mathscr{U}(L) \otimes_{\mathscr{U}(K)} V \cong \operatorname{Hom}_{\mathscr{U}(K)}\left(\mathscr{U}(L),{ }_{\alpha} V\right),
$$

we have $H^{n}\left(L, \operatorname{Ind}_{K}(V)\right)=H^{n}\left(K,{ }_{\alpha} V\right)$ for every $n \geq 0$. Now condition (a) implies $\left({ }_{\alpha} V\right)^{L_{0}}=(0)$. Consequently, (3.4)(2) along with (b) forces the vanishing of $H^{n}\left(K,{ }_{\alpha} V\right)$ for every $n \geq 0$.

\section{REFERENCES}

1. R. Farnsteiner, Lie theoretic methods in cohomology theory, Lie Algebras, (Madison, 1987) (G. Benkart and J. M. Osborn, eds.), Lecture Notes in Math., vol. 1373, Springer-Verlag, Berlin, 1986, pp. 93-110.

2. __ On the cohomology of ring extensions, Adv. in Math. 87 (1991), 42-70.

3. R. Farnsteiner and H. Strade, Shapiro's Lemma and its consequences in the cohomology theory of modular Lie algebras, Math. Z. 206 (1991), 153-168.

4. K. Hirata, On relative homological algebra of Frobenius extensions, Nagoya Math. J. 15 (1959), 17-28.

5. G. P. Hochschild, Relative homological algebra, Trans. Amer. Math. Soc. 82 (1956), 246269.

6. _ـ Semisimplicity of 2-graded Lie algebras, Illinois J. Math. 20 (1976), 107-123.

7. N. Jacobson, Lie algebras, Wiley, 1962; reprint, Dover, 1979.

8. V. G. Kac, Lie superalgebras, Adv. in Math. 26 (1977), 8-96.

9. __ Representations of classical Lie superalgebras, Lecture Notes in Math., vol. 676 (K. Bleuler, H. Pertry, and A. Reetz, eds.), Springer-Verlag, Berlin, 1977, pp. 579-626.

10. F. Kasch, Projektive Frobenius Erweiterungen, Sitzungsber. Heidelb. Akad. Wiss. Math.Natur. Kl. 1960/61, 89-109.

11. __, Dualitätseigenschafter von Frobeniuserweiterungen, Math. Z. 77 (1961), 219-227.

12. J. C. McConnell and J. C. Robson, Noncommutative Noetherian rings, Wiley, 1987.

13. T. Nakayama and T. Tsuzuku, On Frobenius extensions. I, Nagoya Math. J. 17 (1960), 89-110.

14. __, On Frobenius extensions. II, Nagoya Math. J. 19 (1961), 127-148.

15. B. Pareigis, Einige Bemerkungen über Frobeniuserweiterungen, Math. Ann. 153 (1964), 113.

16. G. Shen, Graded modules of graded Lie algebras of Cartan type. II, Sci. Sinica 29 (1986), 1009-1019.

Department of Mathematics, University of Wisconsin-Milwaukee, Milwaukee, WisCONSIN 53201-0413

E-mail address: adbell@archimedes.math.uwm.edu 